\title{
Reusable OIRD Microarray Chips Based on Bienzyme Immobilized Polyaniline Nanowire Forest for Multiplexed Detection of Biological Small Molecules
}

Changxiang Fang, Changyin Zhong, Nan Chen, Lingya Yi, Junying Li, and Weihua $H u^{*}$

Key Laboratory of Luminescent and Real-Time Analytical Chemistry (Southwest University), Ministry of Education, School of Materials and Energy, Southwest University, Chongqing 400715, China; Key Laboratory of Laser Technology and Optoelectronic Functional Materials of Hainan Province, College of Chemistry and Chemical Engineering, Hainan Normal University, Haikou 571158, China.

Email: whhu@swu.edu.cn (W.H.Hu) 

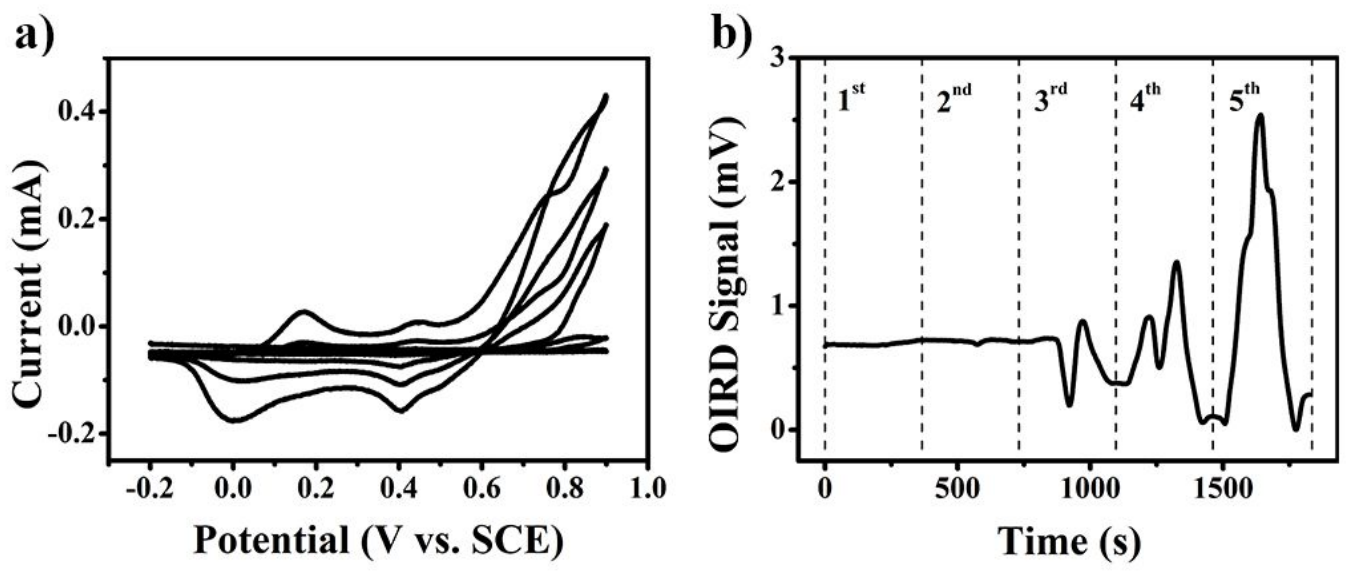

Figure S1. CV curve (a) and in situ OIRD signal response (b) of electrochemical polymerization of polyaniline on FTO.

a)

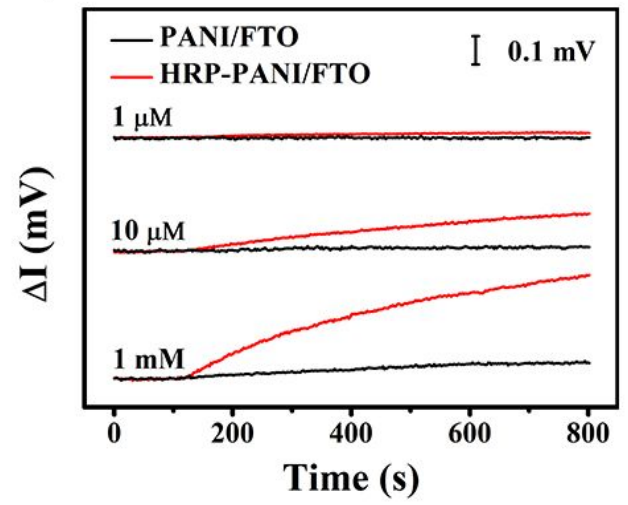

b)

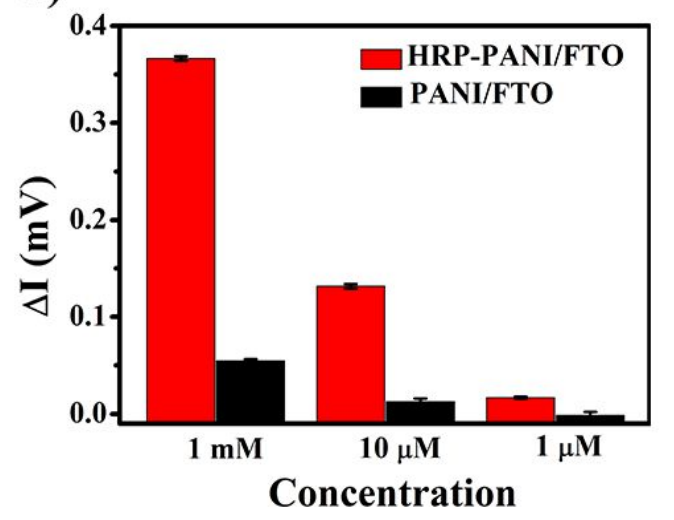

Figure S2. In situ OIRD signal (a) and the OIRD intensity (b) of chip with/without attached HRP for detection of hydrogen peroxide at different concentration.

a)

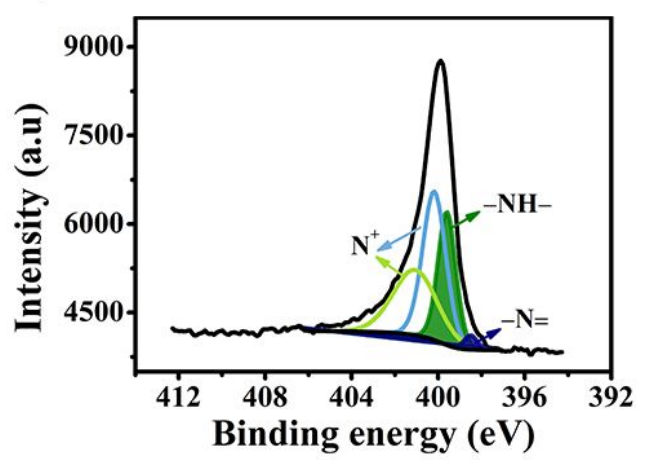

b)

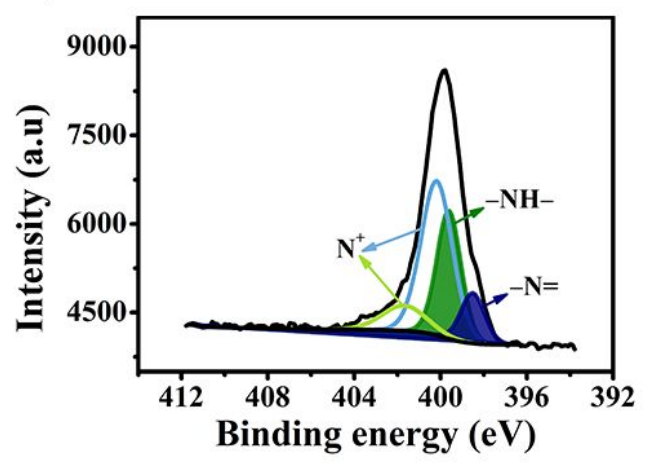

Figure S3. N(1s) XPS spectra of (a) pristine PANI and (b) PANI after the detection of $\mathrm{H}_{2} \mathrm{O}_{2}$. 


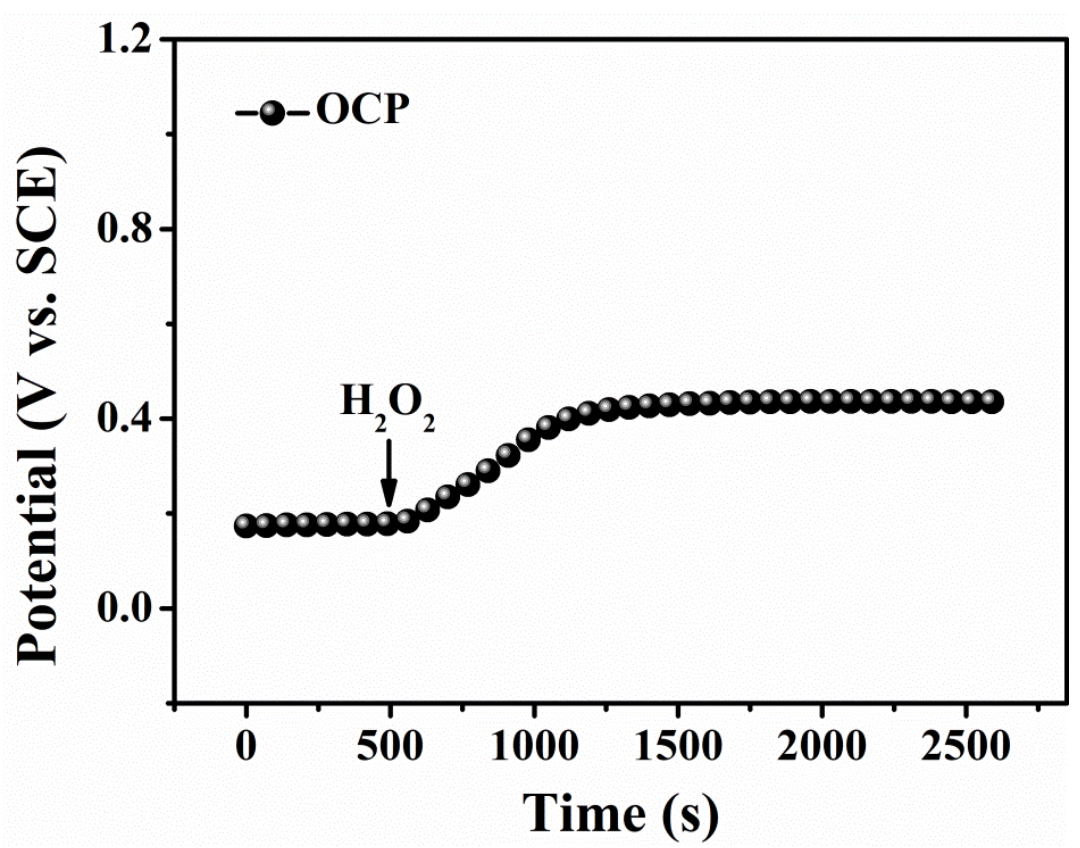

Figure S4. Open circuit potential evolution of the HRP@PANI/FTO chip during the detection of $\mathrm{H}_{2} \mathrm{O}_{2}$ (in $0.01 \mathrm{M}$ of PBS buffer).

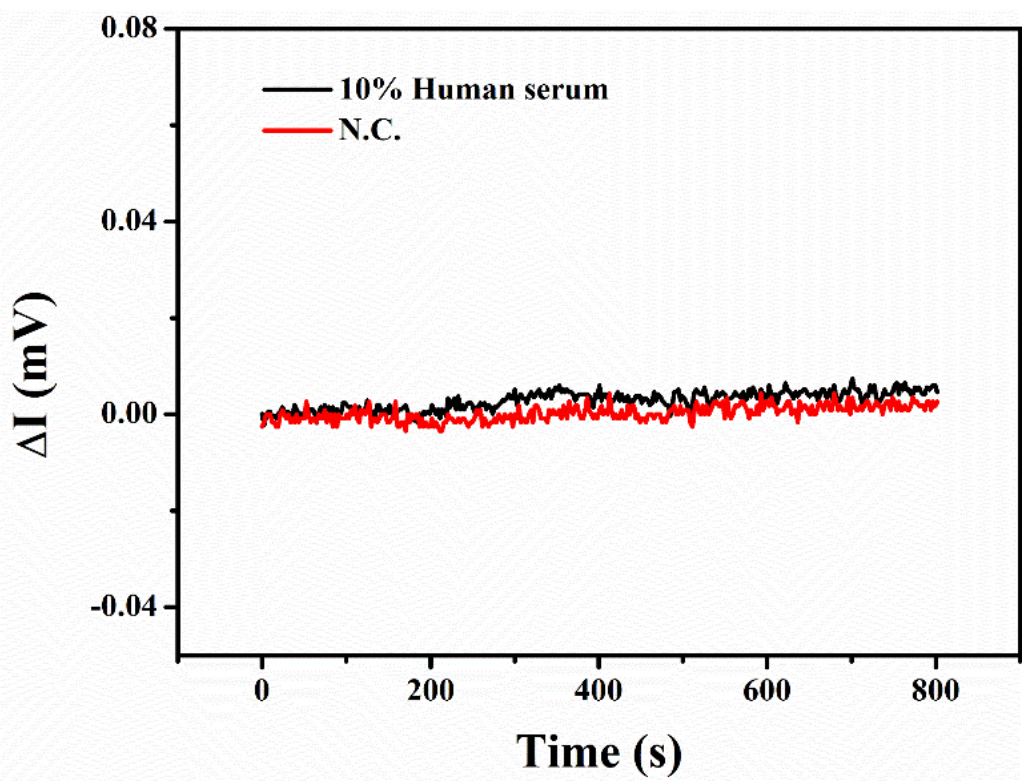

Figure S5. In situ OIRD response of GOD-HRP-PANI/FTO upon the addition of $10 \%$ human serum. N.C. denotes negative control, and was carried out in $0.01 \mathrm{M}$ PBS.

The principle of OIRD. OIRD monitors the interfacial process by measuring the difference between $\mathrm{p}$ and s polarized reflectivity of an elliptically polarized light. In principle, the dielectric constant changes or the thickness/coverage changes caused by 
various reactions at the interface will cause the response of OIRD. The OIRD signal has been mathematically described in the previous work (e.g., Refs 31-37).

The OIRD signal in this work is correlated with the real part of $\Delta_{p}-\Delta_{s}$ :

$$
I_{2 \Omega} \propto \operatorname{Re}\left(\Delta_{p}-\Delta_{S}\right)
$$

Where $\left(\Delta_{p}-\Delta_{s}\right)$ can be described as follow:

$$
\Delta_{p}-\Delta_{s}=\frac{r_{p}-r_{p 0}}{r_{p 0}}-\frac{r_{s}-r_{s 0}}{r_{s 0}}
$$

$r_{p}$ and $r_{s}$ are the $\mathrm{p}$ - and s- polarized reflectivity at the interface after detection, while the $r_{p 0}$ and $r_{s 0}$ are the p- and s-polarized reflectivity at the interface before detection. In the present case, the oxidation of PANI film leads to the change of its conjugate structure, conductivity and doping state, and this, of cause associated with the change of dielectric constant, can be detected by the OIRD. 